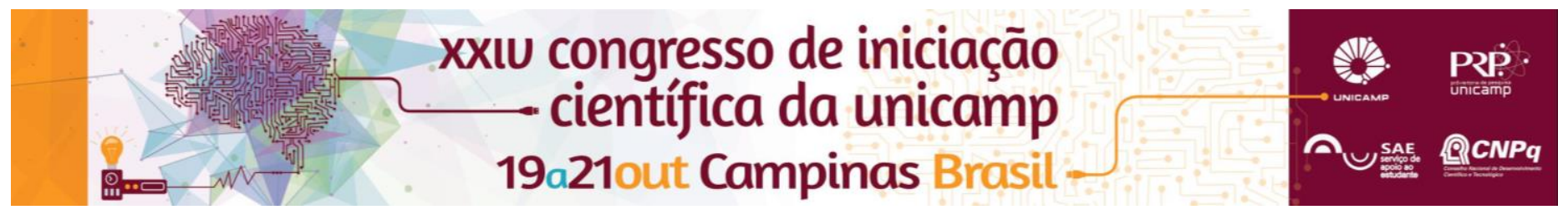

\title{
Role of ARHGAP21 on cell proliferation in C57BL/6 mouse islets
}

\author{
Elis G. Azevedo* 1, Gabriela M. Soares 1, Matheus D. Terrazam 1, Sara T. O. Saad 2, Antonio C. Boschero 1, \\ Helena C. Barbosa-Sampaio 1 \\ 1 Department of Structural and Functional Biology, Biology Institute, University of Campinas, São Paulo, Brazil \\ 2 Haematology Centre, School of Medicine, University of Campinas- UNICAMP, Campinas, Brazil
}

\section{Abstract}

ARHGAP21 participates in cell proliferation, but the mechanisms involved in the role of that GAP are not elucidated. This project aims to investigate the possible involvement of ARHGAP21 in beta cell proliferation mechanisms in pancreatic islet in response to insulin resistance in ARGAP21 haplodeficient mice, after administration of a high fat diet. This project contributes to understanding the cellular mechanisms that lead beta cell mass and proliferation in insulin resistance.

\section{Key words:}

ARHGAP21, cell proliferation, pancreatic islet

\section{Introduction}

Beta cell proliferation is an important compensatory mechanism in response to peripheral insulin resistance, increasing the size of the pancreatic islet. ARHGAP21 regulates GTPases activity and participates in cytoskeletal dynamics, cell cycle progression, transcriptional regulation, cell survival and vesicle trafficking ${ }^{1,2}$, however its mechanisms in pancreatic islets are unknown. This study aims to investigate the possible involvement of ARHGAP21 in cell proliferation in pancreatic islet cells in the compensatory mechanisms during the high fat diet (HFD) treatment, using the ARHGAP21 haplodeficient mouse (HET), compared to C57BL/6 mouse control (CTL).

\section{Results and Discussion}

HET mouse islets express, approximately, $50 \%$ less ARHGAP21 gene

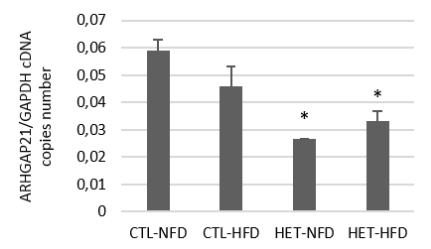

Figure 1: ARHGAP21 gene expression in mice islets. Bars represent average \pm SEM, $\mathrm{n}=2-8,{ }^{*}$ means significantly differences compared to the respective controls, $p<0,05$

As expected, HET mouse islets presented less ARHGAP21 gene content, compared to control, in NFD and HFD. 12 weeks HFD treatment induced no significant decrease in ARHGAP21 gene expression in mouse islets.

\section{ARHGAP21 haplodeficience reduces insulin, glucagon and cyclinD1 gene expression in HFD- treated mouse islets}
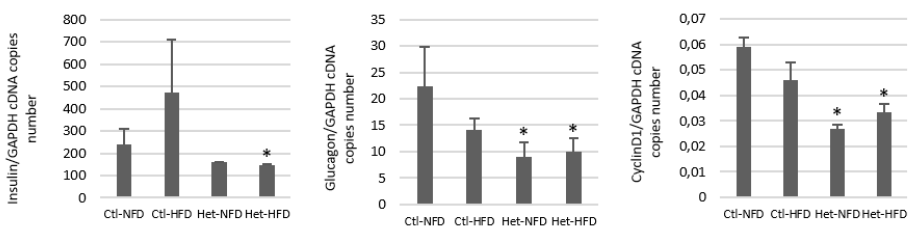

Figure 2: Insulin, glucagon and cyclinD2 gene expression in mice groups. Bars represent average $\pm S E M, n=2-8,{ }^{*}$ means significantly difference compared to the respective controls, $p<0,05$.

\section{ARHGAP21 haplodeficience impairs beta and alpha cell expansion in HFD-treated mice}

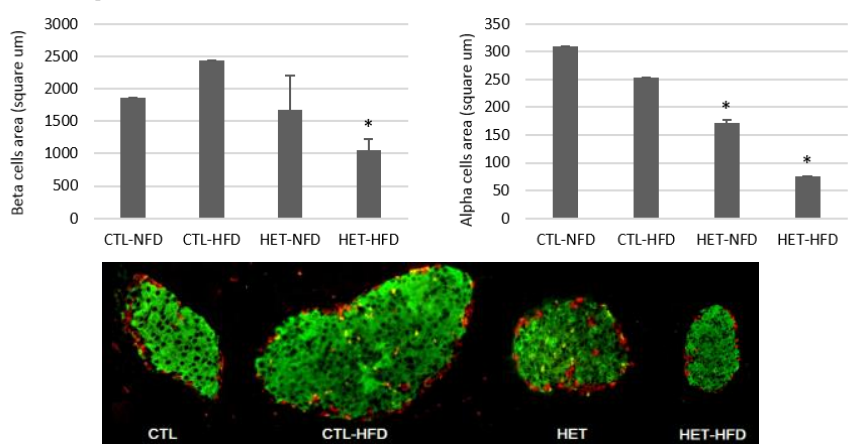

Figure 3: Beta (up left) and alpha cells (up right) areas, expressed in square um in mice pancreas sections. Immunofluorescence representation (center, down) of beta (green) and alpha (red) cells in pancreas sections. Bars represent average $\pm S E M, n=2$, * means significantly differences compared to the respective controls, $p<0,05$.

Reduced insulin gene expression in HET-HFD corroborates with previous results, in which glucose stimulated-insulin secretion in isolated islets is also reduced. Indeed, HET mice are not able to expand beta and alpha cells in response to a HFD, as indicated by cyclinD1 reduced expression and cells areas. These results suggest a ARHGAP21 role in mechanisms involved in islets response to a nutritional excess.

\section{Conclusions}

Our results indicate that ARHGAP21 is essential for beta and alpha cells modulation in response to a nutrient overload in mouse pancreatic islets.

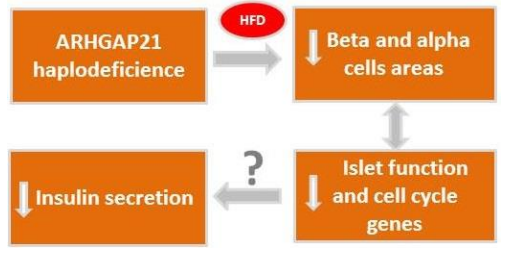

Acknowledgement

Este projeto foi financiado pela Fundação de Amparo à Pesquisa do Estado de São Paulo (Fapesp).

Bolsa de Iniciação Científica: Pibic (CEUA: 3965-1)

${ }^{1}$ Vega F \& Redley A. FEBS Letters 2008, 582, 2093.

${ }^{2}$ Lazarini M et al. Biochimica et Biophysica Acta 2013, 1832, 365. 